\title{
LA PANDEMIA COMO EXPERIENCIA EDUCACIONAL: algunas reflexiones sobre la escolaridad y su estudio
}

\author{
Daniel Johnson Mardones \\ Universidad de Chile, Chile
}

\begin{abstract}
Resumen
“Asistimos a la mayor crisis humanitaria desde la II Guerra mundial" afirmaba en 2015 un informe de UNESCO refiriéndose a la situación de 60 millones de desplazados por causa de conflictos armados, desastres naturales o proyectos de infraestructura. El informe, titulado Educación en emergencias y crisis, constataba que los desastres de la sociedad amplían las brechas de desigualdad. Dicha constatación resonaba con otros documentos que a nivel global habían comenzado ya desde hace más de una década a hablar de educación en situaciones de emergencia. Con todo, la situación a que se vieron sometidos las sociedades y sus sistemas escolares como causa de la emergencia sanitaria desatada por el COVID-19 llevo esta excepcionalidad de la emergencia a ser la situación general, aunque con matices, de toda la humanidad en todos los lugares del planeta. Una situación que, en lo que respecta a los sistemas escolares, marcó una suspensión del tiempo y los espacios escolares a la vez que un esfuerzo por mantenerlos. El presente texto desarrolla algunas ideas que pueden ayudarnos a pensar mejor el presente comprendiendo la pandemia como experiencia educacional y como alegoría de procesos más permanentes de los sistemas escolares, la educación, y las sociedades en general.
\end{abstract}

Palabras clave: Pandemia, Sistemas Escolares, Educación

\begin{abstract}
"We are witnessing the greatest humanitarian crisis since World War II," stated in 2015 UNESCO report referring to the situation of 60 million displaced by armed conflicts, natural disasters or infrastructure projects. The report, entitled Education in emergencies and crises, found that disasters in society widen inequality gaps. This finding resonated with other documents that at a global level had already begun for more than a decade to talk about education in emergency situations. However, the situation to which societies and their school systems were subjected as a cause of the health emergency unleashed by COVID-19 led this exceptionality of the emergency to be the general situation, although with nuances, of all humanity in all the places of the planet. A situation that, in regards to school systems, marked a suspension of school time and spaces as well as an effort to maintain them. This text develops some ideas that can help us to better think about the present, understanding the pandemic as an educational experience and as an allegory of more permanent processes in school systems, education, and societies in general.
\end{abstract}

Keywords: Pandemic, Schooling, Education

ISSN 1645-1384 (online) www.curriculosemfronteiras.org 


\section{La experiencia y la crítica de la escolaridad}

En la actualidad, cualquier discurso sobre la experiencia debe partir de la constatación de que ya no es algo realizable. Pues, así como fue privado de su biografía, al hombre contemporáneo se le ha expropiado su experiencia: más bien la incapacidad de tener y transmitir experiencias quizás sea uno de los pocos datos ciertos de que dispone sobre sí mismo. (Agamben, 2003, p. 7)

Así comienza Giorgo Agamben su texto Infancia e Historia (2003). El sujeto "moderno vuelve a la noche a su casa extenuado por un fárrago de acontecimientos -divertidos o tediosos, insólitos o comunes, atroces o placenteros- sin que ninguno de ellos se haya convertido en experiencia" (p. 8). En efecto, la imposibilidad de la experiencia constituye un aspecto fundamental de la crítica a la modernidad. Más aún, Agamben insiste, "la expropiación de la experiencia estaba implícita en el proyecto fundamental de la ciencia moderna (2003, p. 13). ¿Es de alguna manera la actual pandemia una oportunidad, un paréntesis, o un lugar-tiempo, donde la experiencia ha vuelto a ser posible, ciertamente no de la forma que hubiésemos esperado, y por lo mismo relativiza la expropiación de la experiencia legitimando también su saber? Y, por otra parte, ¿Es esta condición de la experiencia, imposible y expropiada, aplicable a nuestra comprensión de la educación, y específicamente de la escolaridad? Y, de ser así ¿es posible pensar en la educación en tiempos de pandemia, también, como una oportunidad, un paréntesis, un tiempo-lugar, en el que la experiencia vuelve a ser posible en nuestra escolaridad y donde la experiencia es reapropiada en nuestra concepción y trasmisión de los saberes más valiosos? ¿Es una posibilidad de experimentar una educación más allá de la escolaridad moderna, y por lo mismo colonial? ¿O se trata, por el contrario, de un despliegue de la escolaridad moderna-colonial al territorio de lo cotidiano? ¿Una posibilidad de desescolarización de la educación o una expansión de la escolaridad al espacio social más íntimo? Se trata, entonces, más bien de una colonización del interior social, del espacio y el tiempo más próximo a nuestra materialidad corporal, o la posibilidad de una mayor autonomía personal y control del contenido de la propia educación, de la propia vivencia de la escolaridad.

La idea de experiencia ha ocupado un rol central en la crítica de la modernidad (ADORNO, 1967; BENJAMÍN, 2004; HORKHEIMER; ADORNO,1972; JAY, 2005), como la idea de curriculum vivido (AOKI, 2005; PINAR, 2011, 2015) lo ha hecho en la crítica de la escolaridad, y no solo en el contexto angloamericano con el cual probablemente asociamos en primera instancia la palabra experiencia, dado lo que algunos autores han llamado "el culto americano [estadounidense, deberíamos decir] de la experiencia" (JAY, 2005, p. ix). Con todo, podemos encontrar la misma la palabra experiencia usada en la crítica de la cultura moderna por autores como Adorno, para quien en el mundo moderno "la propia posibilidad de la experiencia está en peligro." En su teoría de la seudo-cultura, o mejor traducida de la seudo-formación, Adorno (1959) afirma que los "sistemas delirantes colectivos de la seudo-formación cultural componen lo incompatible: pronuncian el enajenamiento y le sancionan como si fuese un oscuro misterio y trae un sustitutivo de 
experiencia, mentiroso y aparentemente próximo, en lugar de la experiencia destruida (p. 30). Por su parte, Walter Benjamín, escribiendo unas décadas antes que Adorno, se lamenta de la "pobreza de la experiencia humana" en la cual la modernidad ha sumido a la humanidad. La cultura, Benjamín (2004) escribe, que pareciera entonces "algo cosificado. Su historia no sería nada más que el poso formado por momentos memorables a los que no ha rozado en la consciencia de los hombres ni una sola experiencia auténtica, esto es política (p. 135)." El traspaso de la experiencia mediante la escolaridad, esa experiencia de los mayores de que Benjamín nos habla, ha hecho imposible la experiencia de las nuevas generaciones, la que es negada e impedida por la misma. El curriculum entonces, en tanto contendido de la escolaridad niega la experiencia. Y por el mismo, agregaría Freire (1996), la educación moderna parece demasiado alejada de "la emoción del "saber de la experiencia vivida", de los sueños, de la claridad, de las dudas, de la ingenuidad." (p. 65) De esta manera, para los autores citados, la idea de diseñar la experiencia hace imposible el acceso a ella dada la imposibilidad de conocer directamente esta.

De esta la imposibilidad de diseñar la experiencia, incognoscible por definición, llevara a ciertos estudiosos del Curriculum a definir este, el curriculum, como el estudio de la experiencia educacional, cuyo contenido solo se hace posible una vez que se había convertido en pasado, en experiencia vivida. Un estudio que al revés de la ingeniería social, y su obsesión con el diseño, que se enfoca en el futuro, negando la experiencia, mira hacia el pasado. Los puntos solo se conectan hacia atrás. La consecuencia de la definición del contenido de la escolaridad como experiencia vivida, llevaría a la necesidad de pensar un método que hiciera posible su estudio. Esta convicción, producto de la imaginación educacional, adelanto al desarrollo de los enfoques biográficos en otros campos disciplinarios en casi 3 décadas. Entre ellos, en lo que compete a los enfoques biográficos en investigación cualitativa a la autoenografía en sus variadas versiones, la mayoría aún demasiado blancas, la investigación narrativa y otras formas de auto-narrativas que marcaron el giro biográfico. No olvidemos que muchos de estos enfoques al igual que el proyecto interpretativo en investigación cualitativa se definieron como el estudio de la experiencia.

Es este estudio de la experiencia que, acumulada en acervos de conocimiento, y consensuada como legitima en procesos no libres de dinámicas de poder, se organiza en aquello que llamamos disciplinas académicas y disciplinas escolares. Nervi (2004) sostiene que los saberes "entendidos como la organización de la experiencia humana, se ordenan históricamente en disciplinas y estas constituyen específicos y diferenciados campos simbólicos que intentan explicar y comprender, a su vez, específicamente y diferenciados ámbitos de la realidad a través de un discurso y de una práctica de legitimación teóricamente legitimada" (p. 21). La experiencia humana, organizada en acerbos de conocimiento, es ofrecida a las nuevas generaciones a través de la educación formando entonces parte de su propia experiencia humana. Paradójicamente, como han sugerido Cannadine, Keating y Sheldon (2011), es muy poco lo que sabemos acerca de cómo las disciplinas académicas, experiencia acumulada, son experimentadas por quienes las enseñan, las estudian y las profesan. Volviendo a Benjamin: “¿Para qué valen los bienes [de la educación si no nos une a ellos la experiencia? (p. 168). La pregunta se mantiene, adquiere nueva relevancia y se 
complejiza en la actual situación donde el sistema escolar busca mantenerse en funcionamiento al tiempo que la condiciones lo hacen imposible en los mismos términos.

\section{La pandemia como experiencia}

En el movimiento de comprender las tensiones que se establecen en este campo de fuerza acerca de la experiencia de la escolaridad de conservación y discontinuidad, abierta por la pandemia del COVID 19, la noción de inoperatividad, de zonas de indistinción, de indiferenciación, de indeterminación de Agamben (2003) aparece como de gran potencial. En efecto, para Agamben la posibilidad de superar el pensamiento binario, de búsqueda de ideas claras y distintas, que definen la escolaridad moderna en términos, por ejemplo, de teoría y práctica, público y privado, entrenamiento y formación, inclusión y exclusión, igualdad y desigualdad, libertad y control, se basa en encontrar determinados lugares y momentos en que dichas distinciones se vuelven inoperantes. En efecto es lo que ha realizado como en un verdadero experimento de pensamiento claramente descrito y comunicado en lo que podríamos aún llamar la hiperrealidad de la pandemia. Una escolaridad basada en la distinción del espacio escolar del espacio cotidiano, de la cultura "nacional-global de la cultura local, del tiempo escolar del tiempo personal y comunitario; esa escolaridad ya no fue posible en las condiciones vividas durante la escolaridad vivida en los últimos meses, ya casi un año. De alguna forma, en alguna extensión, la escolaridad colonial-moderna entro en una situación de indistinción, de indiferenciación, indeterminación; dados el cierre de las escuelas, el establecimiento de regímenes de confinamiento posibilidades por el decreto de estados de excepción, y el deseo al mismo tiempo de mantener el funcionamiento de la escuela a pesar de las restricciones materiales de presencia y conexión en la nueva situación. En un sentido parecido, podríamos decir que la vivencia de la escolaridad bajo la crisis sanitaria hace más visible el espacio de no-coincidencia Butler (2007, 2003, 2002) que constituye dicha vivencia. En este sentido, Pinar (2011, p. 18) afirma

Esta no coincidencia estructural es el espacio y el tiempo de la subjetividad. En tal tiempo y espacio, caracterizando el cuerpo y su estar en el mundo, cada cual sabe que se encuentra vivo. Uno se vuelve consciente de uno mismo es una experiencia en curso en toda su multidimensionalidad e inaprehensibilidad (Jay, 2005). Es esta no coincidiencia estructural del cuerpo vivo, tiempo y espacio de la subjetividad, que nos invita a experimentar la experiencia.

Para Pinar es esta no coincidencia la que posibilita autorreflexión, el estudio de sí mismo, y como resultado el conocimiento de uno mismo. De esta manera, la no coincidencia la que hace posible no sólo la crítica de lo que existe sino también la reconstrucción de la realidad en lo que podría ser. Es esto lo que ha sido también expresado como un "tercer espacio" (PINAR 2011; AOKI, 2005), donde la pregunta acerca de cuál conocimiento es el de mayor valor tiene la oportunidad de ser respondida (BIESTA, 2003) en el espacio entre lo público 
y lo privado, lo dado y aquello que aún no es. La idea de curriculum vivido, la experiencia educacional, re-conceptualiza nuestra noción de educación, en tanto experiencia educacional vivida, como un espacio liminal intergeneracional. Un lugar de encuentro o imposición, o ambos. Un espacio en el solo podemos-debemos estar-reconocer en el umbral de la relación intergeneracional admitiendo los límites de nuestros conocimientos y la comunicabilidad de los mismos en tanto experiencia. Es ahí donde se juega el que nuestra experiencia educacional sea vivida como un dialogo o un monologo cultural.

Esta relación liminal en educación es similar a la liminalidad que encontramos en la relacionalidad de la investigación, ya redefinida. Se trata de un estar en un umbral que conecta dos espacios. La relación liminal que posibilita, aunque no asegura, el reconocimiento de las limitaciones en todo conocer. Ni siquiera en la comprensión, ese entender desde dentro, estamos completamente dentro del mundo del otro ni completamente fuera del propio. De ahí que la imposibilidad de diseñar la experiencia, incognoscible por definición, llevara a ciertos estudiosos del Curriculum a definir este como el estudio de la experiencia educacional, cuyo contenido solo se hacía posible una vez que se había convertido en pasado. Un estudio que al revés de la ingeniería social que se enfoca en el futuro, negando la experiencia, mira hacia el pasado. Los puntos solo se conectan hacia atrás. La consecuencia la definición del contenido de la escolaridad como experiencia vivida, llevo a la necesidad de pensar un método que hiciera posible su estudio. Esta convicción, producto de la imaginación educacional y cuyo sitio fue la formación docente, adelanto al desarrollo de los enfoques biográficos en otros campos disciplinarios en casi 3 décadas. Entre ellos, en lo que compete a los enfoques biográficos en investigación cualitativa a la autoenografía en sus variadas versiones, la mayoría aún demasiado blancas, la investigación narrativa y otras formas de auto-narrativas que marcaron el giro biográfico. No olvidemos que muchos de estos enfoques al igual que el proyecto interpretativo en investigación cualitativa se definieron como el estudio de la experiencia. Las constataciones ya hechas nos hablan también de la educación como un campo colonizado, cuya sofisticación e innovación intelectual corre muchas veces la misma suerte de aquellos para quienes en su experiencia histórica la palabra colonización no es una metáfora. Piénsese por ejemplo en los trabajos de Freire, experiencias publicadas años después en sus libros La educación como práctica de libertad (1968) y la Pedagogía del oprimido (1971), que adelantaron por lo menos una década a los de Stenhouse (1971), y cuyo nombre apenas aparece en las descripciones históricas del surgimiento de la investigación acción educacional. Estas tradiciones parecen ser especialmente relevantes en un contexto donde las respuestas en los distintos territorios educacionales difícilmente pueden venir desde fuera y donde quienes trabajan y habitan dichos territorios se ven forzados a pensar una respuesta a una situación incierta.

De la misma manera, la educación en tanto disciplina moderna no está libre del aspecto instrumental expresado claramente en la expresión escolaridad. En ese sentido incluso los discursos educacionales sobre la educación; curriculum, didaktik y pedagogía, en tanto teorías de la escolaridad tienen una cierta propensión al reduccionismo universalizando, estandarizando y normalizando y al mismo tiempo marginalizando y segregando las distinciones lingüísticas, culturales, y raciales. Para Tero Autio $(2010,2014)$ es ahí donde se 
manifiestan las herencias intelectuales de la ilustración que lo llevan a llamar teorías de curriculum, a los estudios curriculares, la didaktik, la pedagogía y las llamadas ciencias de la educación. Todo esto no significa que el proyecto emancipador no este presenta, también como característica de la modernidad, sin embargo reservado para un sujeto blanco, adulto, hombre, y de clase media que despliega su conciencia como conciencia humana. Un provincialismo que aún estamos sufriendo. En palabras de Dussel (1995), ahí se manifiesta la modernidad como deseo emancipatorio y mito sacrificial, que también se manifiesta en la escolaridad moderna y en la crítica a la misma. En general, la palabra que aparece como central en esta crítica es formación. Formación que siempre supone una autoreflexión acerca de sí mismo. Bildung es formación y de esa manera autoformación. Autio explica que la autoformación puede ser entendida como un elemento constitutivo de Bildung. Así, autoformación, un enfoque presente en importantes enfoques de investigación en educación y formación docente, trabajando la conexión entre sujetos y cultura, un trabajo de cultivaciónautocultivacion que no ocurre en aislación. La responsabilidad personal de adquirir una forma implica entonces el cultivo de lo personal que es también social, de esa manera política. Se trata entonces de habitar esa zona intermedia entre lo formativo planificado y lo vivido, un ejercicio no solo complejo sino también complicado en el que la escucha de voces variadas no conduce necesariamente a conclusiones correctas, tampoco permanentes, y donde las verdades se hacen esquivas.

En este sentido la pedagógica latinoamericana y el movimiento de reconceptualización de los estudios curriculares en el contexto angloamericano pueden ser entendidos como una reacción a la estandarización, un movimiento que hoy también en el mundo germano parlante usa el concepto de Bildung. Esta crítica de la modernidad resuena con el concepto de currere en el contexto del curriculum psicologizado (AUTIO, 2014; 2005) del mundo angloamericano, el rechazo del mismo por la pedagógica latinoamericana. Finalmente, la palabra formación eco esa preocupación por la hegemonía de la ingeniería social en el mundo educacional. Formación es una de las principales traducciones del concepto alemán de Bildung, lo que hace Autio vincular a la reconceptualización y el trabajo de William Pinar a esta tradición, que de acuerdo a Daniel Throler, es generalmente usada para oponerse a la estandarización educacional bajo la perspectiva instrumental, y entonces se convierte en un concepto que combate los enfoques utilitarios y pragmáticos, contribuyendo a la crítica de la educación occidentalizada. Este reduccionismo es lo que llevo a Adorno a habla de una educación convertida en mercancía y separada de los asuntos propiamente humanos. Una educación deshumanizadora diría Freire. Esto es lo que Adorno (1959) llamo halfbildung (seudo-educación), una educación en que la experiencia ha sido reemplazada por un estado selectivo, desconectado, intercambiable y efímero de estar informado. Después de Auschwitz, la única educación que tiene sentido es una educación que busque una autorreflexión crítica (ADORNO, 1967). Después de dicha critica la palabra bildung, en tanto remite a la autoformación y despliegue de la conciencia europea, de nuevo adulta, blanca, masculina no parece ser suficiente. Una educación que ayude a prevenir otro Auschwitz no es solo individual sino que requiere la influencia de otros. De lo absolutamente otro, del otro 
más allá de la totalidad europea, y el silencio y escucha de la exterioridad más allá del sí mismo, agregaría la pedagógica latinoamericana.

Estas tradiciones que rescatan e invitan al desarrollo de una reflexión sobre la propia experiencia como proceso formativo y autoformativo, aún con sus límites, parecen proveer importantes conceptos para pensar la pandemia como experiencia educacional. Primero, la pregunta como las posibilidades materiales de mantener el nexo de la escuela con los estudiantes más allá de la presencialidad y cómo esta escolaridad en esta zona de indeterminación podía y era deseable que se diera en las condiciones de confinamiento que han caracterizado estos meses de pandemia. Segundo la pregunta de cómo esta experiencia nos ha educado, cual ha sido nuestra experiencia educacional de la escolaridad indeterminada vivida en estos meses; y los que aún nos quedan por delante. A la pregunta curricular, qué conocimiento es el más valioso de ser parte de esta escolaridad en suspenso por la crisis sanitaria, se suma la otra acerca de cómo se ha vivido esta experiencia educacional. Ambas preguntas requieren conversación, también intergeneracional, acerca de nuestra experiencia educacional.

\section{La pandemia como alegoría}

Boaventura de Souza Santos (2020) ha afirmado que el "significado literal de la pandemia de coronavirus es el miedo caótico generalizado y la muerte sin fronteras causados por un enemigo invisible. Pero lo que expresa es mucho más que eso" (p. 32). De esta manera, la situación de pandemia "no es una situación de crisis claramente opuesta a una situación de crisis" (p. 19). La crisis, de esta forma, ha sido una situación normal para sectores importantes de las sociedades y sociedades a nivel global. Una "crisis permanente" cuya causa se relaciona con procesos de larga duración que se actualizan desde los años ochenta con la implementación global, aunque diferenciada, de políticas neoliberales. Esta situación de crisis permanente, prosigue nuestro autor, es

un oxímoron, ya que, en el sentido etimológico, la crisis es, por naturaleza, excepcional y temporal, y constituye una oportunidad de superación para originar un mejor estado de cosas. Por otro lado, cuando la crisis es pasajera, debe explicarse por los factores que la provocan. Sin embargo, cuando se vuelve permanente, la crisis se convierte en la causa que explica todo lo demás. [...] El objetivo de la crisis permanente no se debe resolver. Pero ¿cuál es el propósito de este objetivo? básicamente, hay dos: legitimar la escandalosa concentración de riqueza y boicotear medidas efectivas para prevenir una inminente catástrofe ecológica. (SANTOS, 2020, p. 20)

La Pandemia es así un problema político, o más bien es política. Agamben (2020) nos habla en este sentido de la etimología del término (demos en su significado de cuerpo político) enfatizando que la pandemia se convierte en el nuevo terreno de la política "o de la no política" dado que pareciera ser que todas "Todas las naciones y todos los pueblos están 
ahora permanentemente en guerra consigo mismos, porque el invisible y escurridizo enemigo con el que están luchando está dentro de nosotros" (AGAMBEN, 2020, p. 53). Es en ese sentido, en el que Agamben se pregunta por el futuro de nuevos dispositivos que pueden estarse constituyendo en este contexto. Dispositivos, por cierto, una de cuyas características es "la incapacidad de observarlos más allá del contexto inmediato en el que parecen funcionar" (p. 54). El miedo como estrategia política pareciera posibilitar la imposición de restricciones de las libertades con relativa facilidad. Un nuevo, o no tanto, dispositivo de bioseguridad en desarrollo basado en recomendaciones de la "religión médica" que se suma a los mandatos de la religión del mercado. En este sentido, si la crisis se considera permanente es posible también pensar que el estado de excepción pudiera convertirse en permanente. Alegóricamente, las referencias a crisis sanitarias, situación de crisis, estado de excepción, fe en la solución tecnológica de los problemas sociales, son características que resuenan con las primeras décadas del siglo XX. Un momento que dio lugar al establecimiento de regímenes autoritarios y que coincide, además, con el desarrollo de los sistemas escolares y de salud; lo que Dussel (1984) llama los sistemas pedagógicos del Estado.

Alegoría ha sido también un término aludido por Pinar $(2010,2015,2019)$ para pensar los estudios curriculares introduciendo complejidad temporal en el presente. Para Pinar (2015), la alegoría "atestigua el hecho de que "ahora" es temporal y de múltiples capas, que nuestra salida de este momento presente es inmanente a él, si, es decir, nuestra experiencia es educativa" (p. 15). Una forma de pensar "en la que el sentido aparente de una cosa o texto se ve como una señal algún otro sentido, posiblemente muy diferente" Pinar, 2015, p. 21) y cuyo sentido etimológico, hablar públicamente en una asamblea, "destaca su carácter pedagógico y comunicativo" (p. 27). Este hablar públicamente reunido, se cuenta una historia específica que insinúa un significado y cuyos personajes son a la vez particulares y simbólicos. Para Pinar, la comprensión del curriculum, "incorpora alegóricamente y conscientemente el pasado en el presente, a través de la subjetividad de uno" (PINAR, 2015, p. 27). Esta tipo reflexión sobre la experiencia educacional, que es el curriculum, resulta particularmente relevante en el contexto de pandemia dado que se trata de una forma de pensar un momento histórico de cómo algo nos afecta aunque no podamos determinar su significado, continua Pinar refriéndose a Benjamin. Cómo responder a las preguntas curriculares acerca del conocimiento más valioso y como hemos vivido una experiencia que no entendemos cabalmente. Alegoría parece ser una respuesta posible, dado que ya nos habla de los límites de nuestra comprensión de cualquier experiencia y la complicación que conlleva la conversación en que las respuestas a ambas preguntas pueden tener lugar. Y esto es sobre todo relevante, cuando la escolaridad en suspensión hace aún más visible que la educación es siempre un espacio liminal entre dos generaciones y que cualquier respuesta a las grandes preguntas requieren incorporar al otro educacional en la conversación. Lo que importa es la conversación, Pinar (2015) insiste; y, la alegoría nos lleva a "los estudiantes [...], un destino difícilmente alejado de la provincia del maestro, pero nunca definitivamente dependiente del maestro" (p. 30). Una vez más, el lenguaje de las restricciones se tensiona con el de las posibilidades. 


\section{La escolaridad en pandemia: posibilidades}

Dado que hemos acudido al pensar la escolarización en pandemia como una zona de indeterminación, una escolaridad en suspensión es posible pensar también las posibilidades que esta situación ha conllevado. Estas posibilidades no surgen sólo de la reflexión intelectual sino de un conjunto de experiencias vividas en dicho contexto. En este sentido, Boaventura de Souza Santos (2020, p. 79) nos dice que "L[1]a pandemia y la cuarentena revelan que hay alternativas posibles, que las sociedades se adaptan a nuevas formas de vida cuando es necesario y se trata del bien común. Esta situación es propicia para pensar en alternativas a las formas de vivir, producir, consumir y convivir en los primeros años del siglo XXI".

Una de estas posibilidades, es la centralidad del estudio como quehacer educacional más allá de la concepción de educación bajo la relación enseñanza-aprendizaje, que caracteriza la escolaridad. En este sentido, Pinar (2015) criticando la relación establecida entre curriculum e instrucción, afirma que es el "estudio, no la enseñanza, es el lugar de la educación" (p. 11). Y siga

el estudio se realiza a un ritmo propio y su final desconocido; apoya la reconstrucción subjetiva y social a través del conocimiento académico y la vida cotidiana [...] En contraste con el anonimato y la utilidad de la "información", [...] el conocimiento académico [...] lleva la marca de su compositor, su tiempo y lugar. El conocimiento puede hablar para ti, ya que incorpora la especificidad de sus orígenes, incluso la experiencia vivida de su creador [...] Como forma de testimonio, el estudio alienta historicidad y presagia el futuro. (PINAR, 2015, p. 11)

La palabra estudio; también aludida en el contexto anglosajón para la crítica de la centralidad de enseñanza en educación, y de su expresión en el binomio enseñanzaaprendizaje; es también un concepto usado en la pedagógica latinoamericana para referirse al trabajo intelectual, académico, y de investigación. El estudio escribe Dussel (1974), se opone al olvido como forma de conocimiento produciendo memoria. El estudio escribirá Freire, en su trabajo El acto de estudiar (1965), escrito en su primer año de exilio chileno, requiere una actitud sistemáticamente crítica y una disciplina intelectual se adquiere solamente a través su práctica. El estudio rechaza el consumo de ideas invitando a su recreación, de manera que el estudio de una tradición, o de un linaje académico para usar una la expresión del biólogo Humberto Maturana, implica para Freire el análisis de lo que otro estudiando escribió. El estudiante en tanto lector se convierte en un nuevo escritor; y la escucha del estudiante lo hace un hablante en potencia, diría Benjamin. Estudio dirá Pinar, en tanto forma de vida contribuye a la reconstrucción social y subjetiva tejida a través del conocimiento académico y de la vida cotidiana. Una afirmación que sugiere la posibilidad de conexión entre la oralidad y la escritura, un lugar de encuentro dialógico entre tradiciones 
orales y escritas. Estudio es también entonces una forma de conversación, una palabra que etimológicamente significa transformarse juntos.

Esta posibilidad tensiona el dinámico campo de fuerza abierto por la escuela en suspenso y la profundización de "la barbarie tecnológica que estamos experimentando la cancelación de la vida de cada experiencia de los sentidos y la pérdida de la mirada, permanentemente aprisionada en una pantalla espectral" (AGAMBEN, 2020, p. 60) donde el estudio "como forma de vida" parece al mismo tiempo que recordado como en peligro y que exigen, de acuerdo a Agamben, negarse a la barbarie tecnológica pudiendo así " permanecer viva la palabra del pasado y nacerá — si es que nace - algo así como una nueva cultura" (p. 63). La educación en suspenso profundiza al tiempo que abre posibilidades para que el estudio como acto de memoria pueda restablecer en un posible escenario postpandemia la pregunta por cual es el conocimiento más valioso, reemplazada desde ya décadas por la el despliegue global de la afirmación el conocimiento más valioso es aquel que se evalúa.

\section{Referencias}

ADORNO, T. W. Education after Auschwitz. In: Tiedemann, R. (Ed.). Can one live after Auschwitz? A philosophical reader. Stanford, CA: Stanford University Press, 1967/2003.

ADORNO, T. W. (1959/1980). Theorie der Halbbildung, In: ADORNO, T. W. Gesammelte Schriften Band 8: Soziologische Schriften I, vol. 8, pp. 93-121, 1959.

AGAMBEN, G. ¿En qué punto estamos? La pandemia como política. Quodlibet: Artillería Inmanente, 2020.

AGAMBEN, G. Infancia e historia. Adriana Hidalgo: Buenos Aires, 2003.

AOKI, T. Curriculum in a new key: The collected works of Ted Aoki. Mahwah, N.J.: Lawrence Erlbaum Associates, 2005.

AUTIO. T. The internationalization of curriculum research. In: PINAR, W. F. (Ed.). International handbook of curriculum research. 2 ed. Mahwah, NJ: Lawrence Erlbaum Associates, 2014, pp. 17-31.

AUTIO, T. Globalization, curriculum, and new belongings of subjectivity. In: ROPO, E.; AUTIO, T. (Eds.). International conversations on curriculum studies: Subject, society and curriculum (pp. 122). Rotterdam: Sense Publishers, 2009, pp. 1-22.

AUTIO, T. Subjectivity, curriculum and society: Between and beyond German Didaktik and AngloAmerican curriculum studies. Mahwah, NJ: Lawrence Erlbaum Associates, 2006.

BENJAMIN, W. (2004). Walter Benjamin: Selected writings. Cambridge, MS: Harvard University Press, 2004.

BENJAMIN, W. Illuminations. New York, NY: Schocken Books, 1969.

BIESTA, G. How general can bildung be? Reflections on the future of a modern educational ideal. Journal of Philosophy of Education, n.36, v. 3, 2002, pp.377-390.

BUTLER, J. El género en disputa. Barcelona: Paidós, 2007.

BUTLER, J. Deshacer el género. Barcelona: Paidós, 2006.

BUTLER, J. Cuerpos que importan. Barcelona: Paidós, 2006.

CANNADINE, D.; KEATING, J.; SHELDON, N. The right kind of history: teaching the past in twentiethcentury England. Basingstoke, Hampshire [England]: Palgrave Macmillan, 2011. 
DUSSEL, E. The invention of the Americas: Eclipse of "the other" and the myth of Modernity. New York, NY: Continuum, 1995.

DUSSEL, E. La pedagógica latinoamericana. Bogotá: Editorial Nueva América, 1980.

DUSSEL, E. Para una ética de la liberación latinoamericana. Buenos Aires: Siglo Veintiuno Argentina Editores, 1973.

FREIRE, P. Pedagogy of the oppressed. New York, NY: Herder \& Herder, 1970.

FREIRE, P. La educación como práctica de la libertad. Santiago de Chile: ICIRA, 1968.

HABERMAS, J. The philosophical discourse of modernity: Twelve lectures. Cambridge, MA: MIT Press, 1987.

Hamilton, D. On the origins of the educational terms class and curriculum. In: BAKER, B. (Ed). New curriculum history. Rotterdam: Sense Publishers, 2009, pp. 2-20.

HORKHEIMER, M.; ADORNO, T. W. (1972). Dialectic of enlightenment. New York, NY: Seabury Press, 1972.

ILLICH, I. Deschooling Society. New York, NY: Harper \& Row, 1971.

JAY, M. Songs of Experience: Modern American and European Variations on a Universal Theme. BERKELEY; LOS ANGELES; LONDON: University of California Press, 2005.

JOHNSON-MARDONES, D. Curriculum Studies as an International Conversation: Educational Traditions and Cosmopolitanism in Latin America. New York, NY: Routledge, 2018.

NERVI, M. L. Los saberes de la escuela: Análisis de la renovación disciplinaria en la reforma educacional 1996-2002. Santiago: Editorial Universitaria, 2004.

PINAR, W. F. Educational experience as lived: Knowledge, history, alterity. New York, NY: Palgrave Macmillan, 2015.

PINAR, W. F. (Ed.). International handbook of curriculum research. Mahwah, NJ: Lawrence Erlbaum Associates, 2014.

PINAR, W. F. What is curriculum theory? New York, NY: Routledge, 2012.

PINAR, W. F. The character of curriculum studies: Bildung, Currere, and the recurring question of the subject. New York, NY: Palgrave Macmillan, 2011.

PINAR, W. (2008). Curriculum theory since 1950: Crisis, reconceptualization, internationalization. In: CONNELLY, M; HE, M. F., PHILLION, J. (Eds.). The SAGE handbook of curriculum and instruction. Los Angeles, CA: SAGE Publications, 2008, pp. 491-513.

SOUSA SANTOS, de B. La cruel pedagogía del virus. Buenos Aires: Clacso, 2020.

TRÖHLER, D. (2012). The German idea of Bildung and the Anti-Western Ideology. In: SILJANDER, P.; KIVELÄ, A; SUTINEN. A. (Eds.). Theories of Bildung and growth: Connections and controversies between continental educational thinking and American pragmatism. Rotterdam: Sense Publishers, 2012, pp. 125-134.

\section{Correspondência}

Daniel Johnson Mardones: Possui mestrado em Magister en Educación mención Curriculo y Comunidad Educativa - Universidad de Chile (2010), Ph. D. in Curriculum and Instruction - University of Illinois at Urbana Champaign (2016). Atualmente é académico - Universidad de Chile e Secretario da International Association for the Advancement of Curriculum Studies. Tem experiência na área de Educação, com ênfase em Currículo.

E-mail: djohnson@uchile.cl 
Texto publicado em Currículo sem Fronteiras com autorização do autor 